\title{
Low-Cost Extra-Low Voltage LED Dimmer for Tuneable White Lighting
}

\author{
Roman Hrbac ${ }^{1}$, Tomas Mlcak ${ }^{1}$, Vaclav Kolar ${ }^{1}$, Tomas Vantuch ${ }^{2}$ \\ ${ }^{I}$ Department of Electrical Engineering and Computer Science, VSB-Technical University of Ostrava, \\ 17. listopadu 15, Ostrava, Czech Republic \\ ${ }^{2}$ Centre ENET, VSB-Technical University of Ostrava, \\ 17. listopadu 15, Ostrava, Czech Republic \\ roman.hrbac@vsb.cz.
}

\begin{abstract}
The colour appearance of light sources has a major impact on how people perceive space. With LED illumination lighting, the colour output can be tuned to virtually any perceivable colour, including any hue of white light. It has been established that the right shade of white light during the 24-hour day cycle has a positive effect on the human body's biological system. For this reason, it is very important to focus on the development and application of new lighting systems that have dynamically variable light outputs in order to imitate the sunlight. Circadian regulation is most responsive to short-wavelength light $(460 \mathrm{~nm}=$ blue colour $)$.

This paper describes the development of a low-cost extra-low voltage LED dimmer for tunable white lighting systems aiming to achieve maximum electrical performance and light-output. This dimming module has two independent LED control channels and allows for a variable colour temperature control from warm white to cold white light. Experimental results of the dimming module efficiency measurements, correlated colour temperature (CCT), colour rendering index (CRI), illuminance and power consumption of the luminaire depending on the duty cycle for different control frequencies $(200 \mathrm{~Hz}, 1.5$ $\mathrm{kHz}, 5 \mathrm{kHz}, 10 \mathrm{kHz}$ and $20 \mathrm{kHz}$ ) are presented. The paper further describes the principle of creating control curves for an independent regulation of correlated colour temperature and illuminance. In the form of a table, the control curve outputs may be uploaded into a microcontroller. The paper details the construction, function and the advantages of this LED dimmer prototype.
\end{abstract}

Index Terms-Building automation; Control Systems; Dimmer; Microcontrollers, Microprocessors, Lighting.

\section{INTRODUCTION}

Artificial lighting has contributed to changes in people's lifestyles. People are more active during the night than they used to be. However, recently research has focused on how unnatural lighting influences the human body. For more details see [1]-[3].

The trend in light development is to approximate the

Manuscript received 3 December, 2018; accepted 28 February, 2019.

This paper was supported by the following projects: LO1404: Sustainable development of ENET Centre; SP2019/159, SP2019/28 and SP2019/65 Students Grant Competition and TACR TH02020191 and TACR TH777911, Czech Republic and the Project LTI17023 "Energy Research and Development Information Centre of the Czech Republic" funded by Ministry of Education, Youth and Sports of the Czech Republic, program INTER-EXCELLENCE, subprogram INTER-INFORM and project TN777901 National Centre of Energy. lighting systems to natural daylight. This is to obtain lights that would be able to dynamically change their illuminance and correlated colour temperature to be as natural as possible. During the day, the colour temperature of sunlight may be changed in dependence on daylight or the activity carried out in the room. At present, three types of temperature changes in LED lights are used: Dim to Warm, Tunable White, and Full-Colour Tunable. Each technology has its advantages and disadvantages as stated in [4].

The technology of mixing white-warm and white-cold light colour (Tunable White) uses two LEDs, one of a whitewarm colour and one of white-cold. Electronics helps to change the temperature of the light output via the reduction of the different LED's intensity.

Chromaticity of lights sources has recently been in the centre of attention of many global companies, such as Tridonic [5], Zumbotel [6], Cree [7], Philips [8], as well as smaller companies [9]. Lights with correlated colour temperature control (tunable white) are equipped with LED chips of varying correlated colour temperature controlled by special power supplies. Such sources are controlled using different user's interface. Some of the most frequent interfaces are DALI, Wi-Fi, ZigBee, Bluetooth, IR/RF controls. The end users use different press-button or visualization touch controls, or mobile applications to control the lights. The correlated colour temperature control options are extended by different producers so that the lights could be used for any everyday activity.

The cost of the tunable white technology lights widely differs in dependence on the principle of the applied solution and producer. A LED bulb with correlated colour temperature control controlled via $\mathrm{Wi}-\mathrm{Fi}$ and mobile application, or with an IR (or RF) remote control costs from EUR 27 to 50. The costs of the lighting devices that may be controlled using the above-mentioned control systems range from EUR 80 to 200, while complete system installations are more expensive.

A number of articles dealing with the efficiency of various LED ballasts and analysing data to evaluate the efficiency of the lighting dimming process at different dimming levels and [10] were published.

This paper deals with the development of a low-cost extra-low voltage LED dimmer for tunable white lighting 
systems that allow users to adjust the illuminance and correlated colour temperature. The main goal was to reach the best electrical parameters, light-output and lowest prices using commercially available components. This paper builds on the electrical experiment results published in [11]. Moreover, this paper describes the practical implementation of a LED dimmer prototype, including the measured lighting characteristics in dependence on the electrical properties of the dimmer.

\section{ThE PRINCIPLES OF DIMMING CONTROLS FOR LED LIGHTING}

There are two dimming methods for constant-current LED dimmers: pulse-width modulation (PWM) and constant current reduction (CCR).

\section{A. Design of the PWM LED Dimmer Prototype}

The simplified block diagram of the PWM LED dimmer prototype design for tunable white lighting systems is shown in Fig. 1.

The LED dimmer consists of the following main components: a power supply (IRM-45-48ST, $45 \mathrm{~W}, 230 \mathrm{~V}$ AC/48 VDC, 0.94 A) [12], STM32L011F4P6 low-cost 32bit microcontroller, two ILD6150 dimming LEDs drivers, VS1838B IR receiver sensor, RT9068-33GSP voltage regulator, optocouplers and a RS485 serial interface.

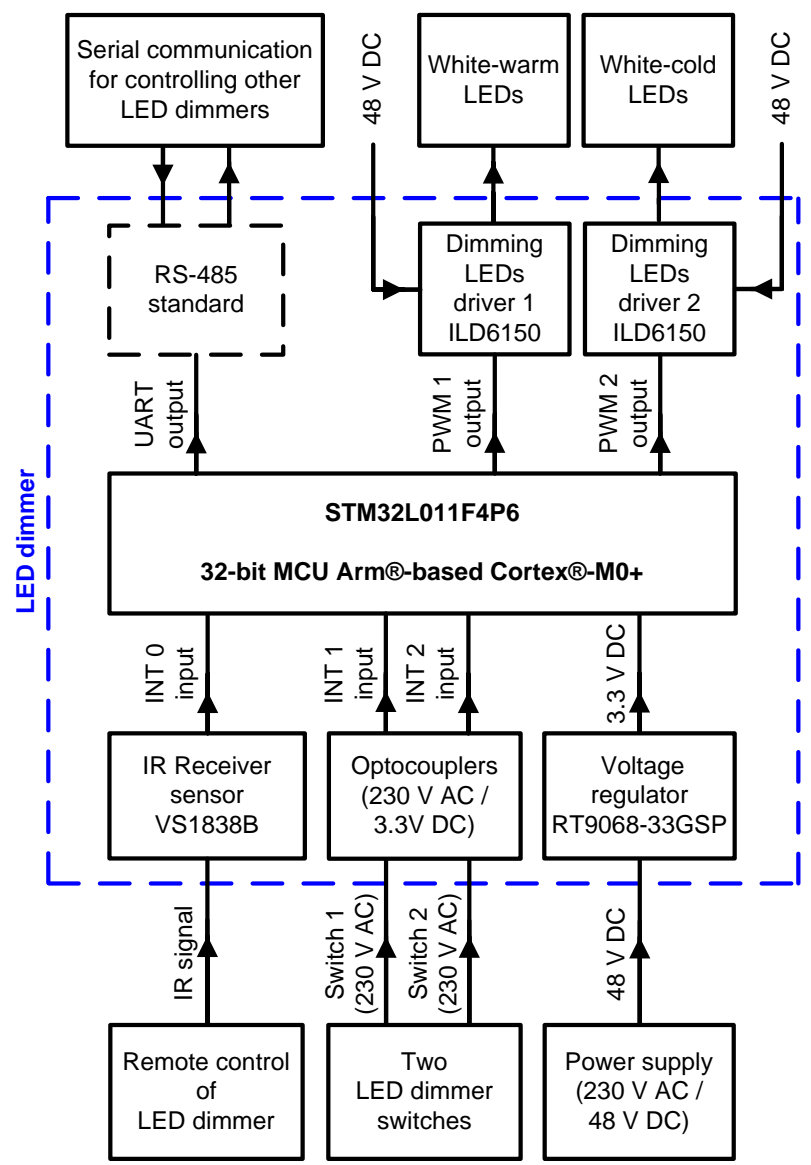

Fig. 1. Simplified block diagram of a LED dimmer design with using STM32L011F4P6 32-bit MCU and ILD6150 drivers.

The STM32L011F4P6 microcontroller controls both the dimming LEDs drivers via PWM signals (PWM 1 and PWM 2). The PWM signals are set by a microcontroller based on its uploaded control characteristics (the control curves for the independent regulation of correlated colour temperature and illuminance) by pressing buttons (switches) directly or using an IR receiver sensor.

Optocouplers function as voltage adjustment, when from $230 \mathrm{~V}$ AC voltage it creates DC voltage and using other DC adjustment elements of $3.3 \mathrm{~V}$, with which the microcontroller may work further. This circuit also carries out the function of galvanic separation of the control circuit from the grid.

Considering a very low consumption $(5 \mathrm{~mA})$ on the $3.3 \mathrm{~V}$ DC, we chose the RT9068-33GSP low dropout (LDO) voltage linear regulator with a wide input voltage range from $4.5 \mathrm{~V}$ to $60 \mathrm{~V}$.

ILD6150 is suitable for LED applications with a wide range of supply voltages from $4.5 \mathrm{~V}$ to $60 \mathrm{~V}$. A multifunctional PWM input signal allows dimming of the LEDs with an analogue DC voltage or an external PWM signal. The efficiency of the LED dimmer is remarkably high, reaching up to $98 \%$ of efficiency over a wide range. For more details, see [11] and [13].

In order to be able to control the luminaries simultaneously, they have to be interconnected via an RS485 output.

On a printed circuit board (PCB) it is possible to set whether the LED dimmer shall work in the Master or Slave mode. LED dimmers in the Slave mode only read the digital values sent by the LED dimmer in the Master mode. This way, identical values of user's illuminance and CCT settings may be ensured. In addition, the LED dimmer in the Master mode may communicate with PLC. Through visualization LED dimmers may be accessed remotely and set up - see Fig. 2.

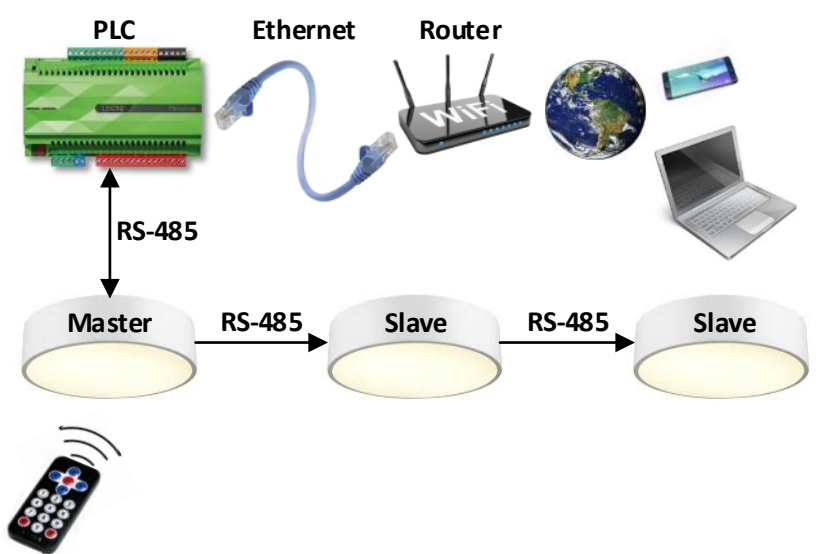

Fig. 2. Practical example of a LED dimmer incorporation in luminaries in a smart-controlled house.

To test the functionality of the LED dimmer, we designed the PCB - see Fig. 3 and Section III for further details.

The construction, function and the advantages of this prototype of LED dimmer are:

- High efficiency of the PWM LED dimmer (up to $98 \%$ ). - Use of a single commercially produced voltage source $230 \mathrm{~V} \mathrm{AC/48} \mathrm{V} \mathrm{DC.} \mathrm{This} \mathrm{source} \mathrm{has} \mathrm{been} \mathrm{subjected} \mathrm{to}$ relevant EMC tests.

- An option to exchange the commercially produced voltage source $230 \mathrm{~V} \mathrm{AC} / 48 \mathrm{~V} \mathrm{DC}$ with a source with better parameters in the future, with no modifications in the LED dimmer. 


\section{EXPERIMENTAL MEASUREMENTS OF LED LUMINAIRE PARAMETERS AT DIFFERENT CONTROL FREQUENCIES}

To measure the control curves we used a spherical integrator and spectrophotometer Jeti specbos 1211, and a function generator MFG2140AF. One channel of the generator connected the dimming circuit for white-warm LEDs, and the second channel connected the dimming circuit for the white-cold LEDs. The control frequency of $1.5 \mathrm{kHz}$ was selected for Pulse-Frequency Modulation.

\section{A. LED Luminaire under Measurements}

The LED panel of the measured luminaire include 112 LED diodes, out of which one half (56 LEDs) had CCT of $2700 \mathrm{~K}$ (white-warm LED with $151 \mathrm{~lm} / \mathrm{W}$ ), and the second half had CCT of $5000 \mathrm{~K}$ (white-cold LED with $166 \mathrm{~lm} / \mathrm{W}$ ) - see Fig. 3. Both LED diode types are connected into a serial-parallel configuration with 4 time's parallel connected series of 14 LED diodes. The used LED diodes are by Samsung, type LM561B. The LED diodes have a Zener diode as a protection from polarity reversal. Graphs of spectral irradiance for white-cold and white-warm LEDs are shown on Fig. 4 and Fig. 5.

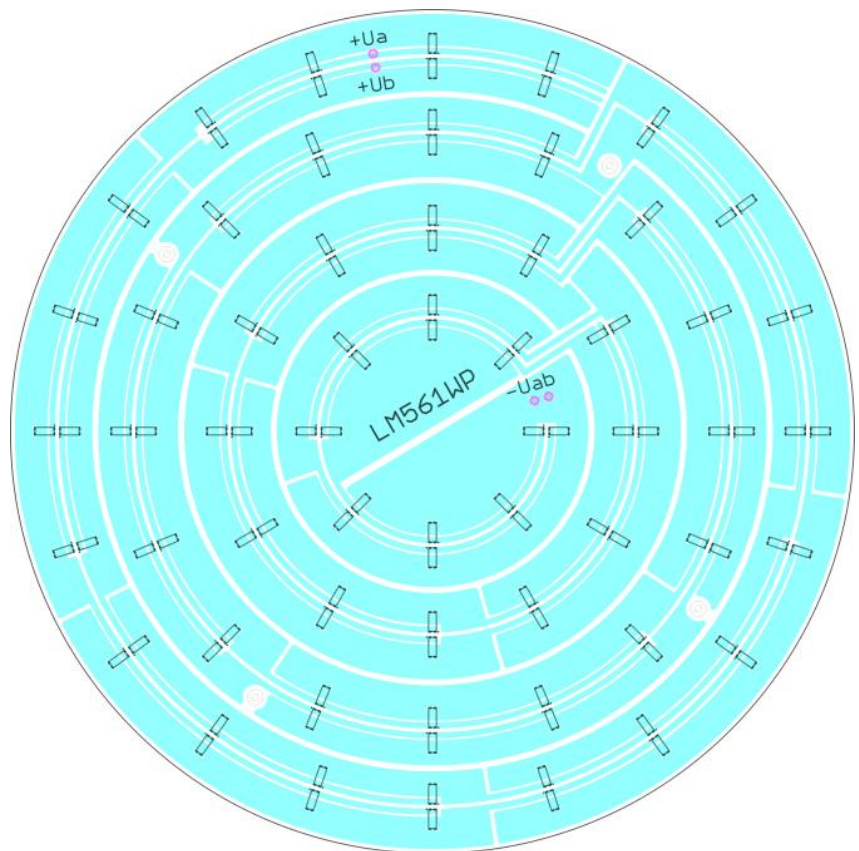

Fig. 3. PCB design of luminaire - top layer.

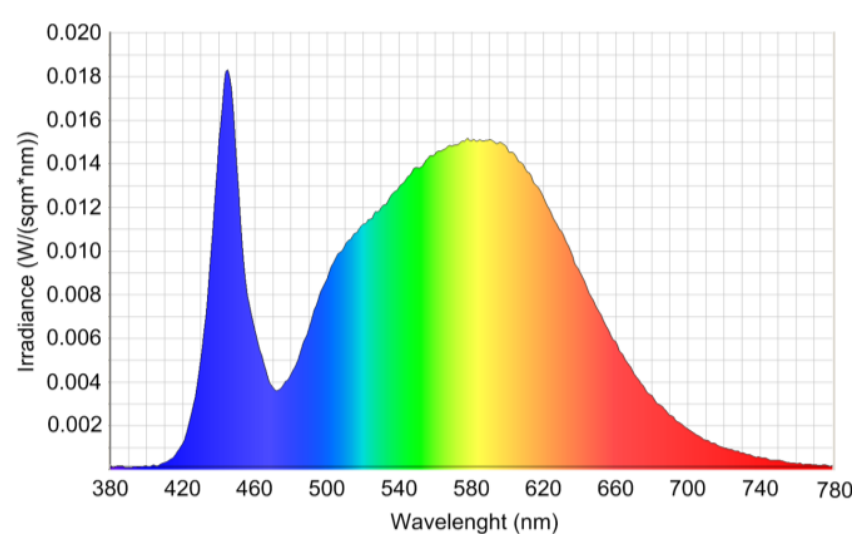

Fig. 4. A graph of spectral irradiance for white-cold LEDs, which illustrates the power contribution from individual wavelength of light.

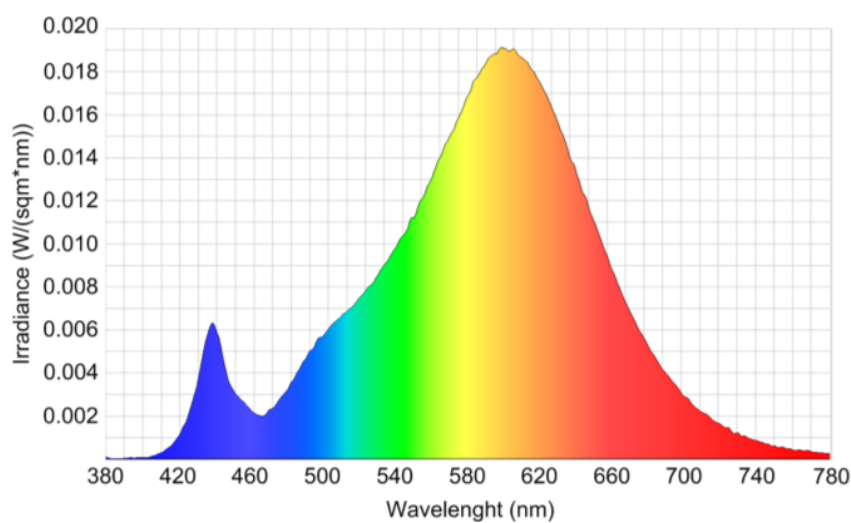

Fig. 5. A graph of spectral irradiance for white-warm LEDs, which illustrates the power contribution from individual wavelength of light.

Using a resistor $R_{\mathrm{SENSE}}$, for more details, see [10], the supply current was set to $370 \mathrm{~mA}$. The current flowing through the discrete LED diodes was $92.5 \mathrm{~mA}$, which corresponds to $138 \%$ of the LED rated luminous flux.

Based on the measured behaviour of dependence of CRI on the duty cycle in Fig. 6 and Fig. 7, it is clear that CRI changes only slightly in dependence on the duty cycle value and the control frequency. A higher decrease in CRI is observed only at lower duty cycle values, when the value of illuminance is low, and at which the spectrophotometer does not work with sufficient precision.

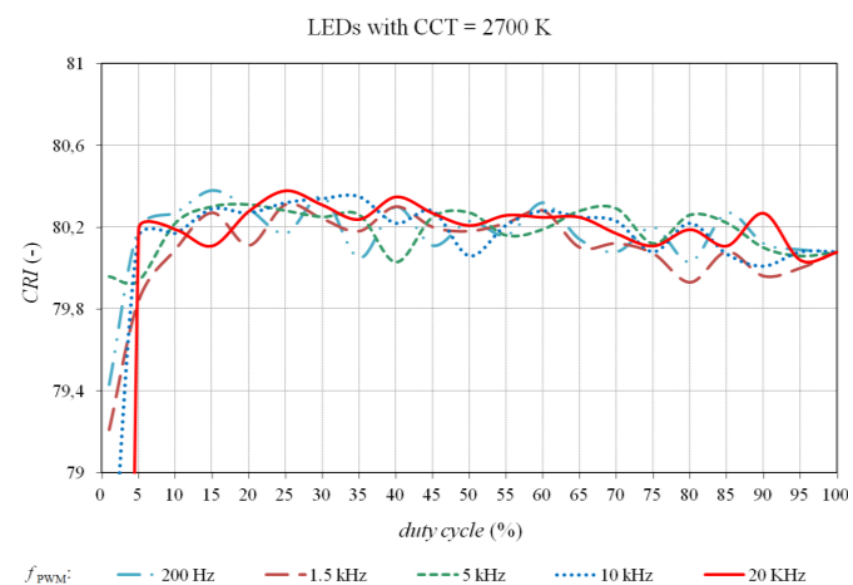

Fig. 6. Dependence of $C R I$ on the duty cycle value under different control frequencies in LEDs with CCT $=2700 \mathrm{~K}$.

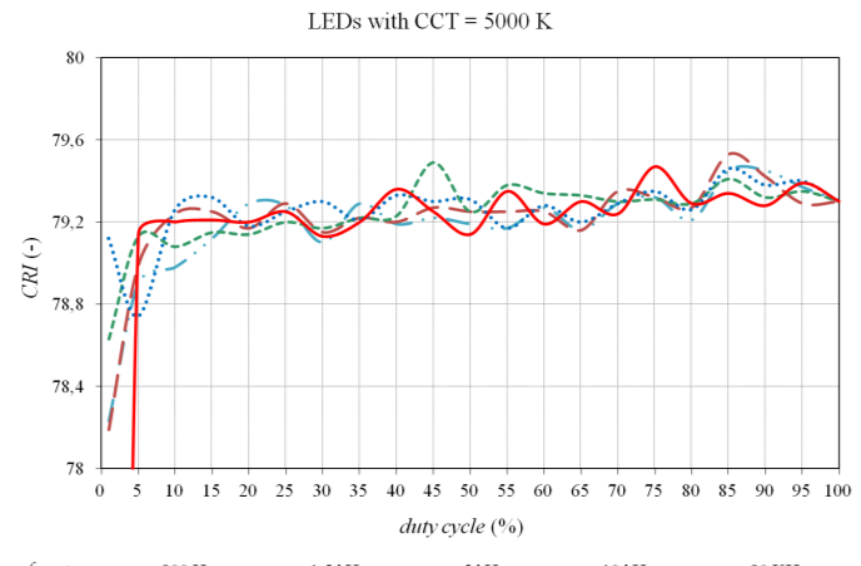

$f_{\text {pwy }} \quad-200 \mathrm{~Hz} \quad--1.5 \mathrm{kHz} \quad---5 \mathrm{kHz} \quad \cdots \cdots 10 \mathrm{kHz} \quad-20 \mathrm{KHz}$

Fig. 7. Dependence of $C R I$ on the duty cycle value under different control frequencies in LEDs with CCT $=5000 \mathrm{~K}$.

Comparing the behaviours in Fig. 8 and Fig. 9 we can see 
that both the LED types are of a high quality and the correlated colour temperature changes only slightly (15 K to $30 \mathrm{~K}$ ) when changing the duty cycle.

Figure 10 clearly implies the difference in CCT under the control frequency of $200 \mathrm{~Hz}$; the decrease is caused by insufficient heating of the measured luminaire as this luminaire was measured first.

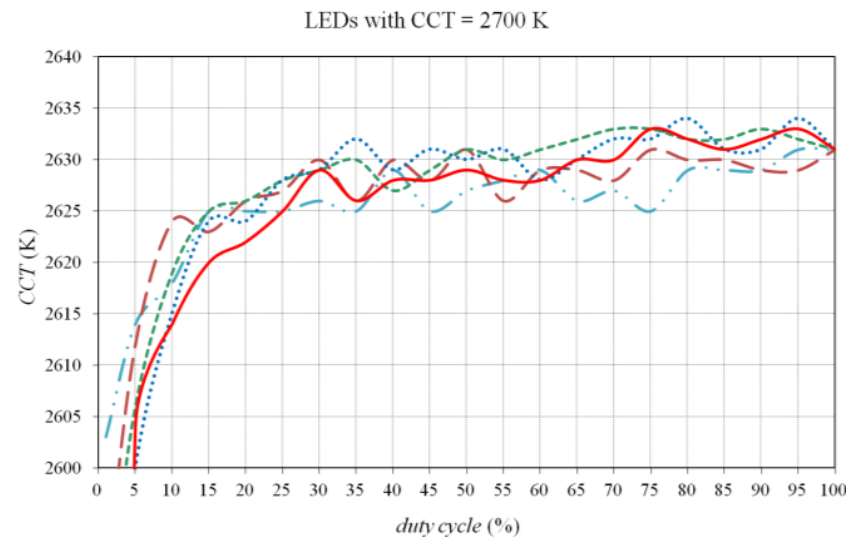

$f_{\text {pwa }}:-200 \mathrm{~Hz} \quad--1.5 \mathrm{kHz} \quad---.5 \mathrm{kHz} \quad \cdots \cdots 10 \mathrm{kHz} \quad-20 \mathrm{KHz}$

Fig. 8. Dependence of $C C T$ on the duty cycle value under different control frequencies in LEDs with CCT $=2700 \mathrm{~K}$.

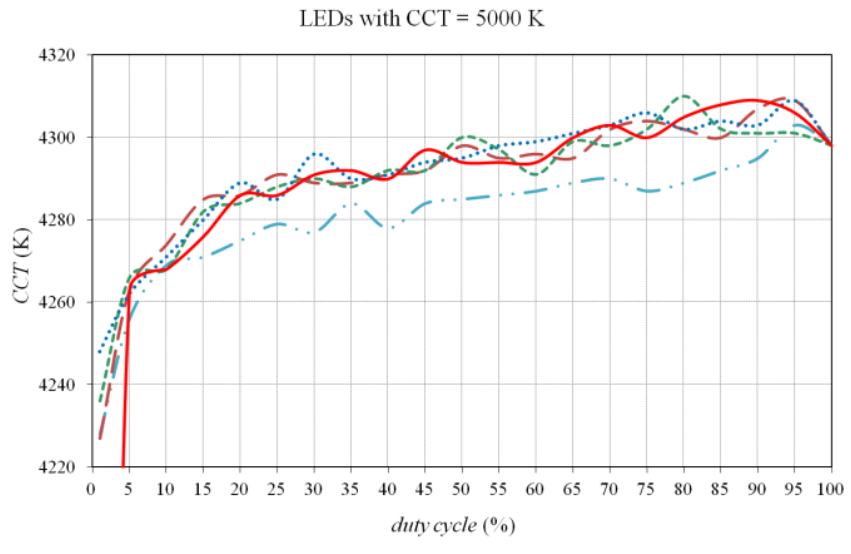

$f_{\text {pwa: }} \quad-.200 \mathrm{~Hz} \quad--1.5 \mathrm{kHz} \quad---\cdot 5 \mathrm{kHz} \quad \cdots \cdots \cdot 10 \mathrm{kHz} \quad-20 \mathrm{KHz}$

Fig. 9. Dependence of $C C T$ on the duty cycle value under different control frequencies in LEDs with CCT $=5000 \mathrm{~K}$.

The measured behaviours in Fig. 10 and Fig. 11 imply how the mean value of the power output supplied into the LED luminaire falls at higher control frequencies.

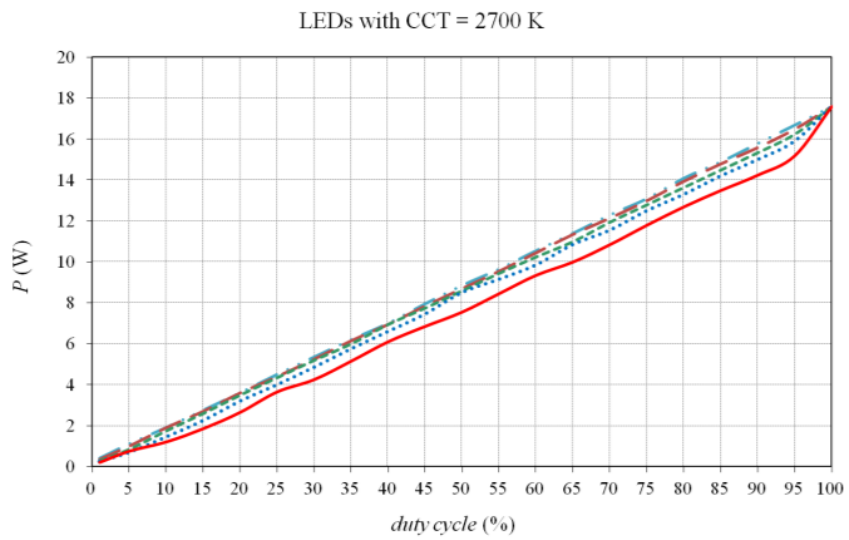

$f_{\text {pwм }}:-200 \mathrm{~Hz} \quad--1.5 \mathrm{kHz} \quad---5 \mathrm{kHz} \quad \cdots \cdots 10 \mathrm{kHz} \quad-20 \mathrm{KHz}$

Fig. 10. Dependence of the power output $P(\mathrm{~W})$ supplied into the LED luminaire on the duty cycle value under different control frequencies in LEDs with $\mathrm{CCT}=2700 \mathrm{~K}$

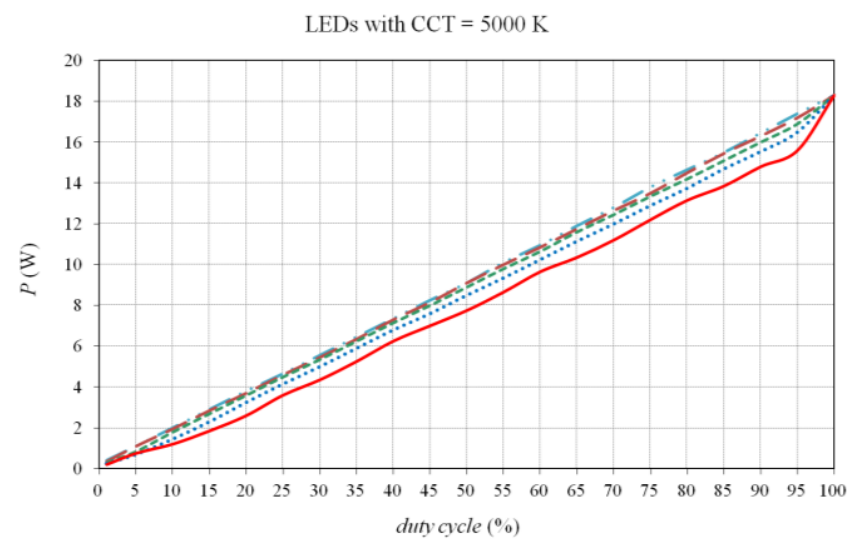

$f_{\mathrm{PWM}^{2}} \quad-.200 \mathrm{~Hz} \quad--1.5 \mathrm{kHz} \quad----5 \mathrm{kHz} \quad \cdots \cdots \cdot 10 \mathrm{kHz} \quad-20 \mathrm{KHz}$

Fig. 11. Dependence of the power output $P(\mathrm{~W})$ supplied into the LED luminaire on the duty cycle value under different control frequencies in LEDs with CCT $=5000 \mathrm{~K}$.

Based on the measured behaviours in Fig. 12 and Fig. 13, we may observe that the control frequencies have identical slopes and that they overlap, which implies that none of the selected control frequency up to $20 \mathrm{kHz}$ causes a perceptible supplementary loss. This way, the specific output remains unchanged.

LEDs with $\mathrm{CCT}=2700 \mathrm{~K}$

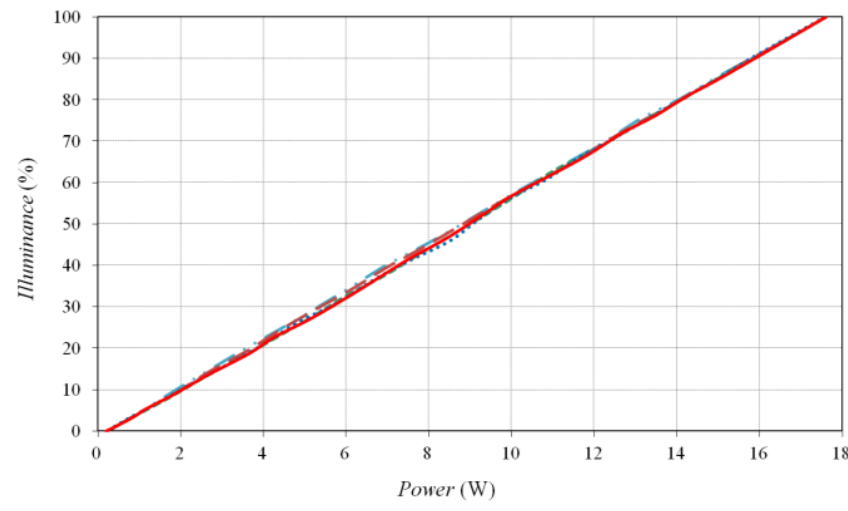

$f_{\mathrm{PWM}}: \quad-.200 \mathrm{~Hz} \quad--1.5 \mathrm{kHz} \quad---5 \mathrm{kHz} \quad \cdots \cdots 10 \mathrm{kHz} \quad-20 \mathrm{kHz}$

Fig. 12. Dependence of illuminance on the power output $P(\mathrm{~W})$ supplied into the LED luminaire under different control frequencies in LEDs with $\mathrm{CCT}=2700 \mathrm{~K}$

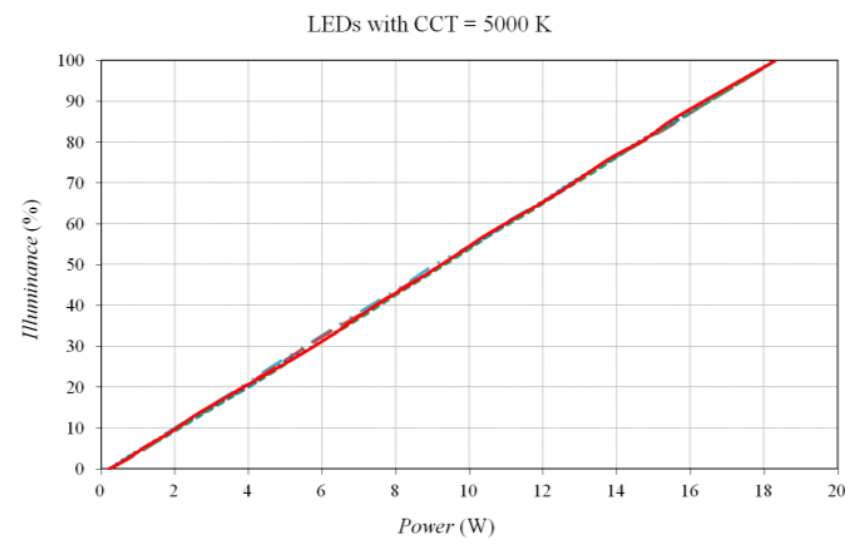

$f_{\mathrm{pwM}}:-200 \mathrm{~Hz} \quad--1.5 \mathrm{kHz} \quad---5 \mathrm{kHz} \quad \cdots \cdots .10 \mathrm{kHz} \quad-20 \mathrm{kHz}$

Fig. 13. Dependence of illuminance on the power output $P(\mathrm{~W})$ supplied into the LED luminaire under different control frequencies in LEDs with $\mathrm{CCT}=5000 \mathrm{~K}$

The experimental measurements proved that under identical duty cycle value a change in the control frequency changes the power output, which alters the illuminance 
proportionally. This implies that once designed control curves are bound onto one control frequency value. In case of a more pronounced change in the control frequency value, new measurements must be carried out and new control curves proposed.

\section{DESIGN AND MEASUREMENTS OF LED LIGHT'S CONTROL CURVES}

\section{A. Measurement of Control Curves Proceeded as Follows}

First, we set the constant value of a duty cycle for whitewarm LEDs to $5 \%$, and we incrementally added $5 \%$ of white-cold LEDs at a time, i.e. from $5 \%$ to $100 \%$. Next, we set $10 \%$ of the duty cycle for white-warm LEDs, and again incrementally added $5 \%$ of white-cold LEDs at a time, i.e. from $5 \%$ to $100 \%$. An increment of $5 \%$ was used to reach $100 \%$ of the duty cycle for white-warm LEDs.

The measurement output was a set of values, using which the required control curves were set up. We assumed that a user would want to set the illuminance and correlated colour temperature independent on one another. Excel by Microsoft was used to construct the control curves. To obtain a high sensitivity of regulation, regulation spacing was from 0 to 100. To obtain the selected spacing, we interpolated the measured values. The resulting values of control curves for constant correlated colour temperature are plotted in Fig. 14.

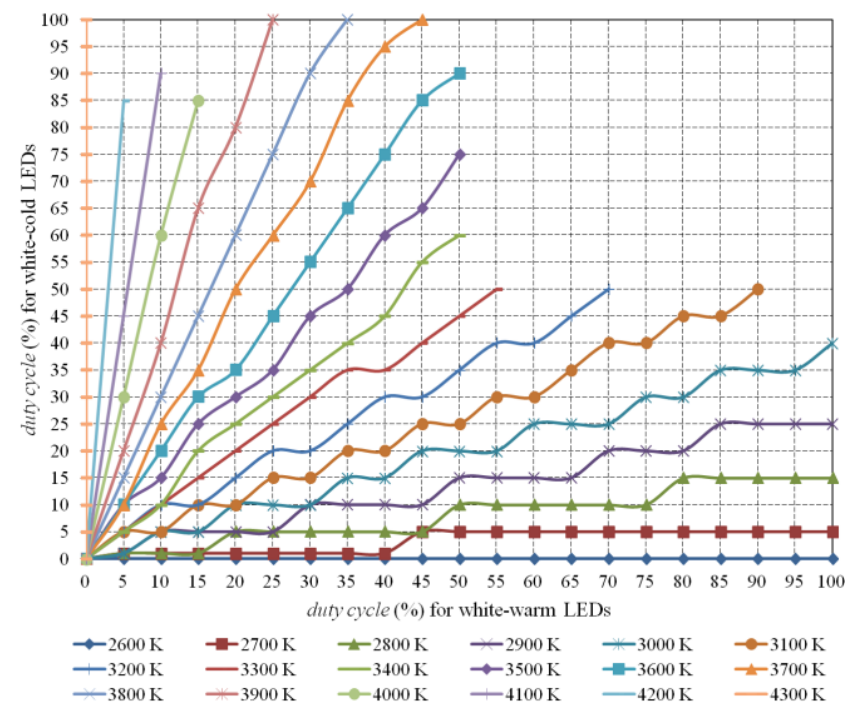

Fig. 14. Control curves for constant correlated colour temperature plotted from the measured values.

In the next step, we linearized the control curves, which helped to remove the inaccuracies arising in connection with the applied method of measurement and search for the nearest measured values. Figure 15 shows the linearized curves for the constant correlated colour temperature and the constant illuminance plotted by means of linear regression formulas. In order to continuously pass between the regulation of correlated colour temperature and illuminance, it is important to find and plot the intersections of the curves in Fig. 16.

\section{B. Control Curves for Independent Regulation of}

Correlated Colour Temperature and Illuminance Plotted by Means of Intersection Points

Excel is not able to recognize whether the plotted curves intersect in the chart or not.

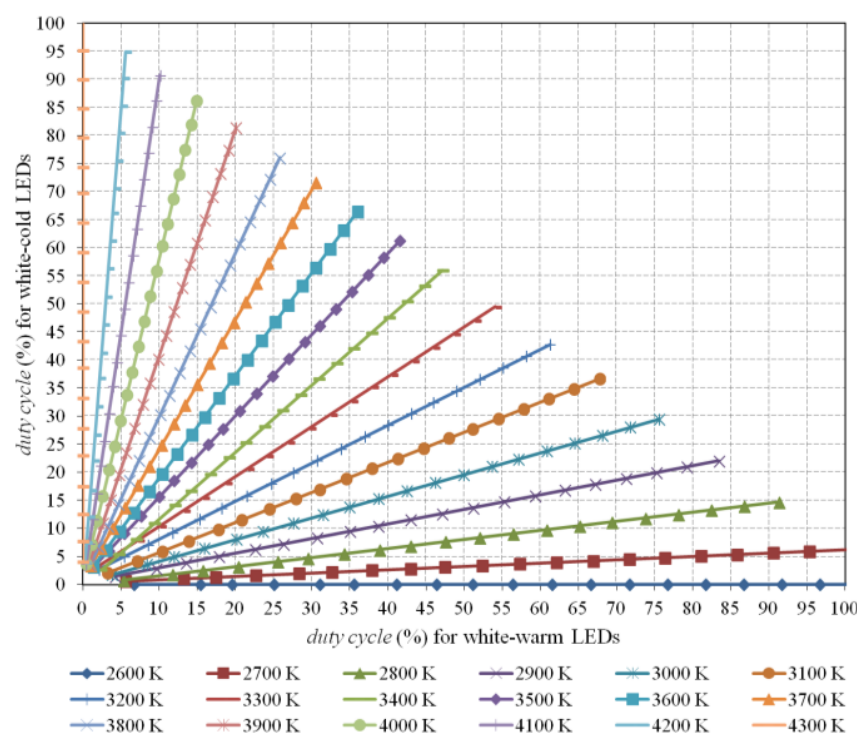

Fig. 15. Control curves for the constant correlated colour temperature plotted by means of intersection pointsUsing the equations, a table is made to plot the curves with intersections, see Fig. 16 and Fig. 17.

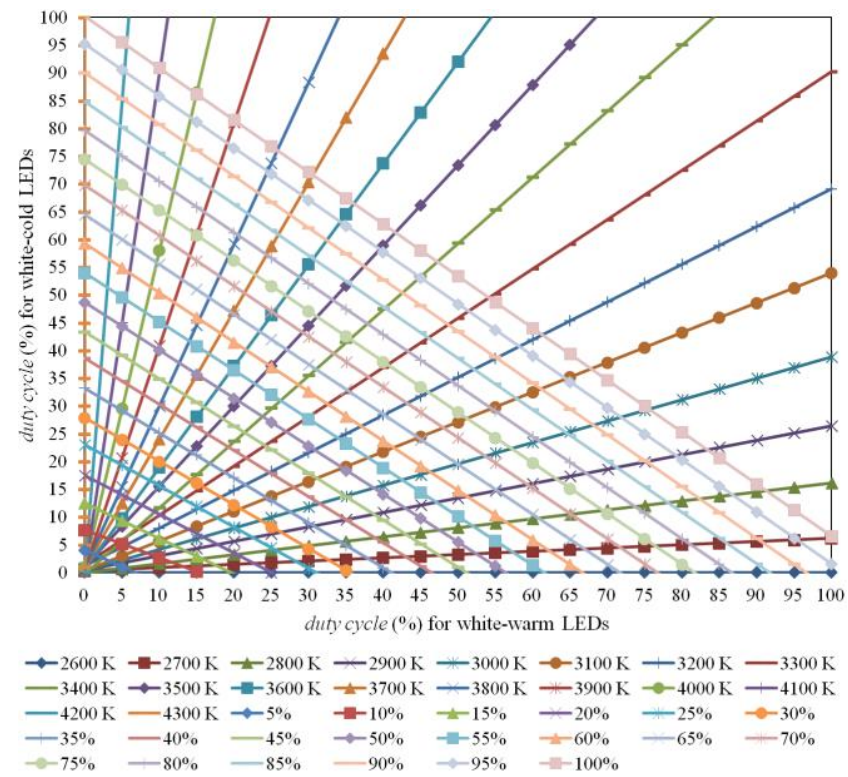

Fig. 16. Combination of both linearized control curves: for constant correlated colour temperature and for constant illuminance.

But as the linearized curves have the shape of lines, they may be described using a line formula (1). This way, it is possible to calculate the intersections of the lines and plot them using Excel. To calculate the intersection, the equations are placed as equal (2):

$$
\begin{gathered}
y_{1}=a_{1} x_{1}+b_{1}, y_{2}=a_{2} x_{2}+b_{2}, \\
a_{1} x+b_{1}=a_{2} x+b_{2}, \\
x=\left(b_{2}-b_{1}\right) /\left(a_{1}-a_{2}\right), \\
y=a x+b .
\end{gathered}
$$

The values $a$ and $b$ may be calculated using functions SLOPE to calculate the value $a$ and INTERCEPT to calculate the value $b$.

The output from Fig. 18 is Table I that may be uploaded into a microcontroller. This way, the control is executed via steps set up at the beginning. The table also shows values in red that exceed $100 \%$. 


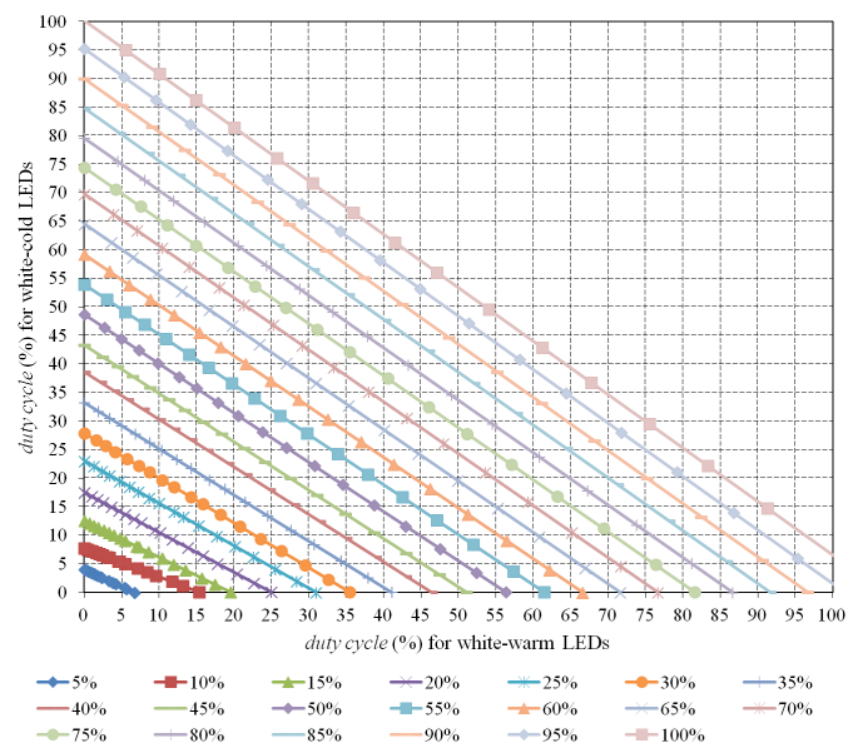

Fig. 17. Control curves for the constant illuminance plotted by means of intersection points.

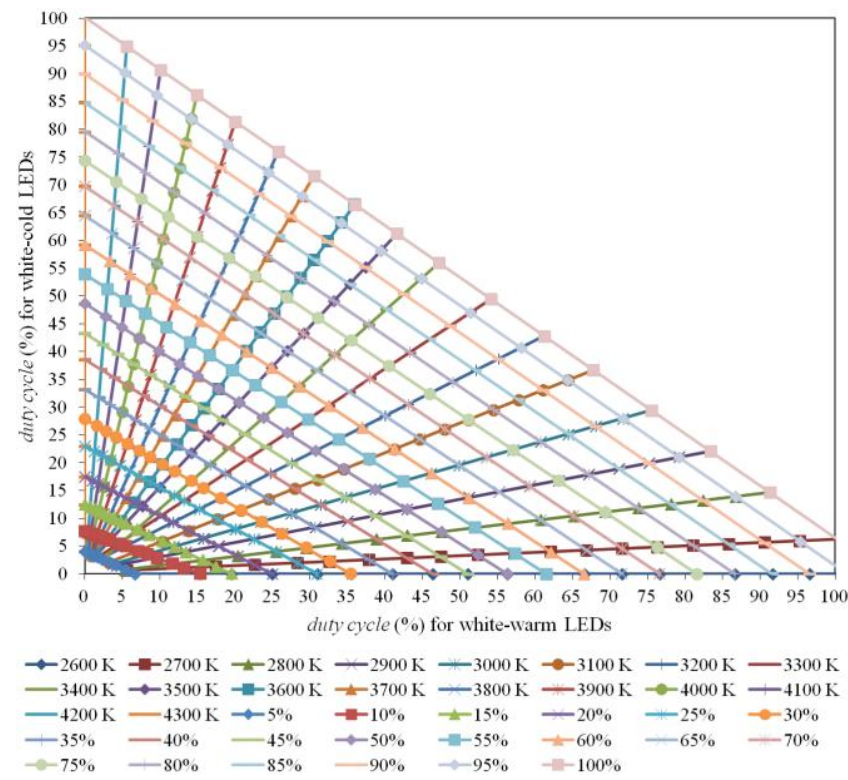

Fig. 18. Final chart of the control curves for the independent regulation of correlated colour temperature and illuminance.

TABLE I. EXAMPLE OF A TABLE WITH FINAL VALUES

\begin{tabular}{|c|c|c|c|c|c|c|c|c|c|c|}
\hline \multicolumn{11}{|c|}{ AVAILABLE FOR A MICROPROCESSOR. } \\
\hline Axis & $\mathrm{x}$ & $\mathrm{y}$ & $\mathrm{x}$ & $\mathrm{y}$ & $\mathrm{x}$ & $\mathrm{y}$ & $\mathrm{x}$ & $\mathrm{y}$ & $\mathrm{x}$ & $\mathrm{y}$ \\
\hline $\begin{array}{l}\text { Duty } \\
\text { cycle }\end{array}$ & \multicolumn{2}{|c|}{$2600 \mathrm{~K}$} & \multicolumn{2}{|c|}{$2700 \mathrm{~K}$} & \multicolumn{2}{|c|}{... } & \multicolumn{2}{|c|}{$4200 \mathrm{~K}$} & \multicolumn{2}{|c|}{$4300 \mathrm{~K}$} \\
\hline $5 \%$ & 6.7 & 0 & 5.6 & 0.6 & $\ldots$ & $\ldots$ & 0.2 & 3.9 & 0 & 4.0 \\
\hline $10 \%$ & 15.3 & 0 & 13.2 & 1.1 & $\ldots$ & $\ldots$ & 0.4 & 7.4 & 0 & 7.7 \\
\hline $15 \%$ & 19.5 & 0 & 17.5 & 1.3 & $\ldots$ & $\ldots$ & 0.7 & 12.0 & 0 & 12.5 \\
\hline $20 \%$ & 25.0 & 0 & 22.7 & 1.6 & $\ldots$ & $\ldots$ & 1.0 & 16.8 & 0 & 17.5 \\
\hline$\cdots$ & $\ldots$ & $\cdots$ & $\ldots$ & $\ldots$ & $\ldots$ & $\cdots$ & $\cdots$ & $\ldots$ & $\ldots$ & $\cdots$ \\
\hline $95 \%$ & 101.6 & 0 & 95.3 & 5.9 & $\ldots$ & $\ldots$ & 5.3 & 90.2 & 0 & 95.2 \\
\hline $100 \%$ & 106.9 & 0 & 100.3 & 6.2 & $\ldots$ & $\ldots$ & 5.6 & 95.0 & 0 & 100.2 \\
\hline
\end{tabular}

This is because white-cold LEDs have a slightly higher specific output than white-warm LEDs, and do not reach the identical value of illuminance at the duty cycle of $100 \%$.

\section{CONCLUSIOnS}

In the modern times, people tend to spend much of their time in enclosed areas. However, the majority of them do not realize that the lack of daylight directly influences their human body and its good functions. As previous studies have shown, cool white light with a high proportion of blue light has a stimulating effect and promotes concentration, whereas warm white light is calming. Tunable white LEDs technology is one way how to face the problem.

The hardware and software presented in this paper can independently control the LED's correlated colour temperature at constant illuminance or the LED's illuminance at constant correlated colour temperature. Both of these options are chosen by each user in 100 discrete steps, with flicker-free quality light down to $1 \%$ dimming level. The control is done using press-button switch or IR receiver sensor.

The principal idea when designing the LED dimmer built on a commercial switching device IRM-45-48ST by MEAN WELL. This device is designed to supply all electronic circuits, including both the LED drivers ILD6150. IRM-4548ST was selected as it has no load power consumption $<0.1 \mathrm{~W}$ and $90.5 \%$ efficiency. The maximum efficiency stated by the ILD6150 LED driver producer reaches $98 \%$.

Based on the experimental measurements, we may claim that the optimum efficiency is obtained under the control frequency of $1.5 \mathrm{kHz}$. This value combines a good efficiency along the dimming range and a low chance of flicker. In this case, starting with the duty cycle > $29 \%$ the overall efficiency of the LED dimmer $>80.5 \%$, the efficiency of ILD6150 > $92.5 \%$, and the efficiency of IRM-45-48ST > $88.5 \%$, both in LEDs with CCT $=2700 \mathrm{~K}$, and LEDs with CCT $5700 \mathrm{~K}$. At the duty cycle $=100 \%$ the overall efficiency of the LED dimmer is $85.5 \%$. It must be pointed out that even the control frequencies below $20 \mathrm{kHz}$ do not cause any supplementary loss, and that specific output remains unchanged - see Fig. 12 and Fig. 13.

The paper also describes the principle of measuring the lighting control curves and their possible modification using Microsoft Excel in order to independently regulate the correlated colour temperature and illuminance. The result of the conversion is a table of values that may be uploaded into a microcontroller STM32L011F4P6.

The experimental waveforms of the prototype were shown to verify the feasibility of the proposed scheme.

\section{REFERENCES}

[1] M. S. Rea, M. G. Figueiro, A. Bierman, J. D. Bullough, "Circadian light", Journal of Circadian Rhythms, vol. 8, p. 2, 2010. DOI: 10.1186/1740-3391-8-2.

[2] C. Rich, T. Longcore, Ecological consequences of artificial night lighting. Los Angeles, USA: Island Press editor, 2006.

[3] K. J. Navara, R. J. Nelson, "The dark side of light at night: physiological, epidemiological, and ecological consequences", Journal of Pineal Research, vol. 43, no. 3, pp. 215-224, 2007. DOI: 10.1111/j.1600-079X.2007.00473.x.

[4] N. Miller, Understanding LED Color-tunable Products. [Online]. Available: https://www.energy.gov/eere/ssl/understanding-led-colortunable-products.

[5] Tridonic. [Online]. Available: http://www.tridonic.com/

[6] Zumbotel. [Online]. Available: http://www.zumtobel.com/ 
[7] Cree. [Online]. Available: http://www.cree.com/

[8] Philips Lightning. [Online]. http://www.usa.lighting.philips.com/

[9] A-LIGHT. [Online]. Available: https://www.e-light.cz/

[10] Z. Slanina, P. Valicek, T. Novak, K. Sokansky, "Interior lightning system sensors placement optimization", in Photonics Applications in Astronomy, Communications, Industry, and High-Energy Physics Experiments, 2018, p. 127. DOI: 10.1117/12.2501559.

[11] R. Hrbac, V. Kolar, T. Novak, L. Prokop, J. Vanus, "Low-cost solution of LED driving, designed for tunable white lighting systems", IFAC-PapersOnLine, vol. 51, no. 6, 2018, pp. 396-401. DOI: 10.1016/j.ifacol.2018.07.186.

[12] IRM-45-48ST. Mean Well. [Online]. Available: https://www.czechmeanwell.cz/meanwell/IRM-45-48ST-Mean-Well-Miniaturni-zdroj-sterminal-45W-48V-d4838.htm

[13] Infineon: ILD6150 - Advanced Thermal Protection for High Power LEDs with 60V LED Driver IC ILD6150, Datasheet PDF, pp. 1-24, Rev 2.2; 3/15, 2015, Infineon Technologies AG, Germany. 